\title{
The Impact of Gender, Avatar and Height in Distance Perception in Virtual Environments
}

\author{
Hugo Coelho ${ }^{1(\bowtie)}$, Miguel Melo ${ }^{2}$, Frederico Branco ${ }^{1,2}$, \\ José Vasconcelos-Raposo ${ }^{1,2}$, and Maximino Bessa ${ }^{1,2}$ \\ 1 University of Trás-os-Montes and Alto Douro, Vila Real, Portugal \\ hcoelho@utad.pt \\ 2 INESC TEC, Porto, Portugal
}

\begin{abstract}
Virtual Reality is becoming more popular over the years because it allows the user to be the main actor in another environment and interact with it in real time. New interaction methods are being studied, like tangible interfaces, but there is little work done related to small distances when grabbing objects through a virtual environment. This study is important because, in our perspective, interaction in virtual reality will be at arms reach, meaning that the user will interact within very close distances (under $1 \mathrm{~m}$ ). In this paper, the research team further evaluate distance perception using gender, the presence of avatar and height (fixed or personalised). The sample consisted of 64 participants (32 females and 32 males) evenly distributed between all four conditions (8 males and 8 females for each condition). Results revealed that gender does have an impact on small distance estimation; height does not have an impact on distance estimation; and avatar does make a difference when trying to grab a real object through the virtual environment.
\end{abstract}

Keywords: Virtual Reality - Distance perception $\cdot$ Gender $\cdot$ Height • Avatar

\section{Introduction}

Virtual Reality (VR) allows the user to play the main role in a virtual environment (VE) and develop the feeling of being there as they interact with the it [1]. Thus, interactions are an important characteristic of VR, and companies are working to implement a truly intuitive and natural interaction [2]. All interaction methodologies can be used to interact with the VE, the only problems are recognising the right movement, the gesture of the action the user wants to perform and syncing them with the VE. There are ways to overcome those problems like tracking real objects or even the user and replicating everything in the VE. One way to do it is by using a tracking system like OptiTrack [3], but even if the object is well placed in the VE, there is no guarantee that the user will reach it when trying to grab the object. [2] said that, of all interaction techniques, direct

(C) Springer Nature Switzerland AG 2019

Á. Rocha et al. (Eds.): WorldCIST'19 2019, AISC 931, pp. 696-705, 2019.

https://doi.org/10.1007/978-3-030-16184-2_66 
picking and direct manipulation are the most natural methods for the user to interact with the VE. Since direct picking and direct manipulation are easier for the user, it is important to understand how people perceive small distances. With this, one can establish how to include objects naturally inside the VE and generate knowledge about small distances.

Since direct picking or manipulation is the most natural methods to interact with the VE, our work aims to study if the presence of an avatar (hand representation present or not) and height (personalised or fixed height) changes the way people perceive virtual objects within small distances. The research team also considered gender as the independent variable in the data was collected by the research team. The remainder of this paper is structured as follows: the second section will present a literature review of distance perception and some concepts. The third section is where the methodology and procedure will be presented. The results are shown in the fourth section, following by the discussion in the fifth section and conclusions on the sixth section.

\section{State-of-the-Art}

Distance perception has been studied using different methods and different variables such as real environment vs VE, depth cues, the presence of avatar, and height. For instance, [4] studied the comparative perception between the real world, a semi-spherical projection and an immersive large screen. Authors concluded that in the real environment people could be precise when estimating distance but in the VE they tend to underestimate distances by $50 \%$. Moreover, [5] performed a study where they shifted the horizon upwards by $11.5^{\circ}$. The shift performed in his experiment did not change the way people perceived distances, and they also concluded that for distances higher than $2.5 \mathrm{~m}$ people tend to overestimate while when presented with distances less than $1.5 \mathrm{~m}$ people tend to underestimate them.

Studies on how the perception of distances changes from the real world to the VE and from interior to exterior in real environments was made by [6]. They concluded that the distance estimation is different comparing the real-world observations and VE observations, but the results obtained from the interior experience are not statistically different from the results obtained in the exterior environment.

There is no consensus about the presence of avatar and distance estimation. Some authors state that the presence of avatar increases the accuracy in distance estimation [7], others say that even a dislocated and/or static avatar increases the accuracy [8] and others state that only a well tracked-avatar leads to an increase of accuracy [9]. However, there are also authors stating that the presence of avatar does not change the way people perceive distances [10].

Regarding the height in the VE, [11] studies if decreasing or increasing the virtual height changes distance perception. The results showed that decreasing the virtual height by $50 \mathrm{~cm}$ had no impact on distance estimation being the explanation that people sometimes have to perform tasks being crouched so they 
are used to being lower than their actual height. On the other hand, increasing the virtual height by $50 \mathrm{~cm}$ decreases the accuracy of distance estimation by $20 \%$.

Studies are showing that females and males see the VE differently; for example, females have a natural experience when presented with the computergenerated environment while males have a more natural experience when presented with the captured content environment [12]. While there are differences in natural experience, studies on distance estimation show that there is no statistical difference when comparing females with males [13].

Besides all the variables described above, there is also work that studied technological variables like Field of View and Head Mount Display (HMD) comparison. These studies are essential since its needed such technologies to see the VE, which influences the user's perception of the VE. [14] studied if the Field of View of the HMD could change the way people perceive distances. They reported that the Field of View does not have an impact on distance estimation. Regarding the state-of-the-art HMD comparison, [15] studied if different HMD could lead to a different distance perception. Their results showed that there is no statistical difference in distance estimation between them.

Distance perception is widely evaluated by using the Blind Walk methodology [16]. In this methodology, the user walks blindfolded to a particular object and stops where he thinks the object is. This approach has some problems since people are hesitant to walk blindfolded, but an acoustic signal or a researcher walking alongside the participant seems to solve that issue. Perceptual correspondence is another technique that can be used to measure distance perception; it uses a reference distance to compare other distances [17]. Verbal reports can also be used, and they measure the distance between the participant and the target object [16]. Time Imagined Walking is a methodology where the user is standing still, blindfolded and uses a chronometer to indicate the time to reach the target object. Using the mean velocity of the participant one can calculate the distance that the participant thought the target object was at [16]. These distance evaluation methodologies can be used to measure two types of distances: Egocentric and Exocentric. Egocentric distance is measured from the feet of the participants to the target object; and Exocentric distance is the distance between two target objects placed in an environment [8]. Usually, both of them are measured using absolute distances, meaning that the participant has to report absolute values about the distance he thinks the target object is at [8].

\section{Methodology}

The adopted methodology consists of a quasi-experimental design (the researcher controls the assignment of the condition the user will perform), cross-sectional study (at a specific point of time) with a quantitative focus. The sampling technique used was the non-probabilistic convenience sampling technique. 


\subsection{Sample}

In this study, the sample consisted of 64 participants (32 males and 32 females) equally distributed in four groups. Their age ranged between 18 and 44 years old $(M=22.22$ and $S D=4.199)$ with all participants reporting normal to corrected-to-normal vision. Also, the samples had heights between 152 and $195 \mathrm{~cm}(M=169.98$ and $S D=11.077)$.

(a)

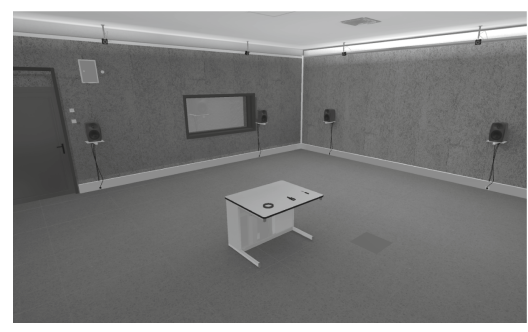

(b)

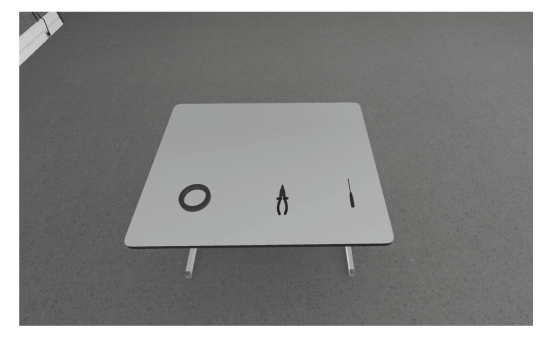

Fig. 1. Virtual representation of the VE. (a) Virtual replica of the experimental room; (b) Representation of the virtual objects.

\subsection{Materials}

To perform the actions of touching a real object throughout an HMD, one created a VE which is a replica of the real room where the experiments took place, Fig. 1a. Also, all three virtual objects and the two avatar hands were replicated to be used in the VE, Fig. 1b. The equipment used to deliver the visual stimulus was the Oculus Rift DK2 HMD. The tracking of the user's head movement and the positions of the plier, tape, and screwdriver was achieved by using the Optitrack's Motive unified motion capture software platform. The application ran on a desktop computer with an Intel i7-5820K, three NVIDIA GTX 980 GPU and $32 \mathrm{~GB}$ of RAM. The Optitrack system was mounted in a room ( 7 by $7 \mathrm{~m}$ ) and consisted of eight cameras, two cameras on each wall, allowing to track objects in an area of 4 by $4 \mathrm{~m}$. To track each object, three or more Optitrack markers per object were used so that the Optitrack software could get the position and rotation of the object, allowing us to reproduce it in the VE.

\subsection{Variables}

In this study, the research team defined three independent variables: Gender (female and male), Height (Fixed or personalised) and Avatar (not present vs present). As for dependent variables, the research team only have the reports saying if the participant touched the real object or not. 


\subsection{Procedure}

Before the user enters the room, the researcher calibrates the room using the OptiTrack system, ensuring that the VE is aligned with the real room. As the participants entered the room, they were asked to sign a consent form and a sociodemographic questionnaire. Next, each participant put the HMD. In the VE, they had the same height as their real one measured using the OptiTrack system. Regardless, the researcher asked the participants if the height felt natural. The researcher changed the height until the participant felt comfortable (increasing or decreasing the virtual height accordingly). After adjusting the virtual height, the researcher chose an object randomly and asked the participant to touch the virtual object and stop where they think the virtual object was. This task was performed one time for each object. When the user's hand stopped, the researcher took notes whether the hand touched the chosen object or not.

\subsection{Statistical Procedures}

Since all the independent variables were dichotomous, the research team did the statistical procedure called Chi-square Test for Association. In this test, all the independent variables have to be nominal or ordinal variables, and one has to have the independence of observations, meaning that there is no relationship between observations. Another requirement is that all expected cell counts have to have a minimum of 5 observations, and if the number is above that, one report the Pearson's chi-square; otherwise, one report the Fisher's exact test.

\section{Results}

Since the research team had three independent variables (female or male, fixed or personalised height and with or without avatar), the first analysis performed was the comparison between gender for each group. A chi-square test for association was conducted between gender and the frequency with which participants placed the hand on top of the objects correctly.

\subsection{Gender Reports}

For all the groups, all expected cell frequencies were greater than five. There was no statistically significant association between gender and the frequency with which participants placed the hand on top of the objects correctly for without an avatar and fixed height condition, $\chi^{2}(1)=1.343, p=0.247$. The same happened for the condition without an avatar and personalised height where we got $\chi^{2}(1)=0.000, p=1.000$.

As for the group with avatar and fixed height, there was a statistically significant association between gender and the frequency with which participants placed the hand on top of the objects correctly for this group, $\chi^{2}(1)=10.243$, 
$p=0.001$. There was a moderate association between gender and the frequency with which participants placed the hand on top of the objects correctly, $\Phi=0.462, p=0.001$.

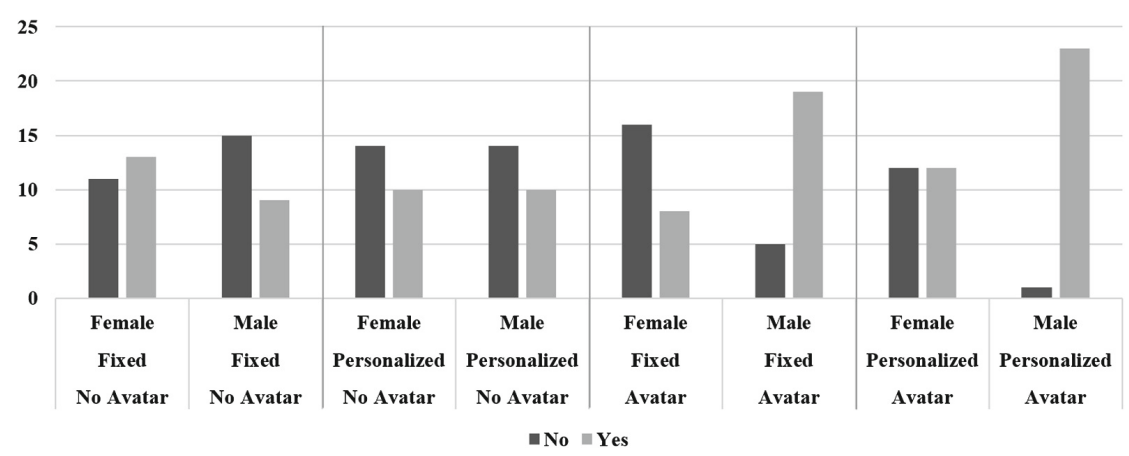

Fig. 2. Gender comparison for all conditions.

For the group with avatar and personalised height, there was a statistically significant association between gender and the frequency with which participants placed the hand on top of the objects correctly for this group, $\chi^{2}(1)=12.765$, $p<0.001$. There was a strong association between gender and the frequency with which participants placed the hand on top of the objects correctly, $\Phi=0.516$, $p<0.001$. The frequencies are shown in Fig. 2 .

\subsection{Height Reports}

After analysing gender variable for each group and realising that gender does make a difference on touching objects through the virtual environment, one analysed the height and avatar variables taking into account the previously reported differences between gender.

For height, a chi-square test for association was conducted between height and the frequency with which participants placed the hand on top of the objects correctly. For the two groups (without avatar group; and with avatar and female participants group), all expected cell frequencies were greater than five. There was no statistically significant association between height and the frequency with which participants placed the hand on top of the objects correctly for the group without an avatar, $\chi^{2}(1)=0.169, p=0.681$. One got the same result for the group with avatar and female participants, $\chi^{2}(1)=1.371, p=0.242$.

As for the group with avatar and male participants, not all expected cell frequencies were greater than five. Therefore a Fisher's Exact test was conducted between the height and the frequency with which participants placed the hand on top of the objects correctly. There was no statistically significant association between height and the frequency with which participants placed the hand on top of the objects correctly, $p=0.188$. The frequencies can be seen in Fig. 3 . 


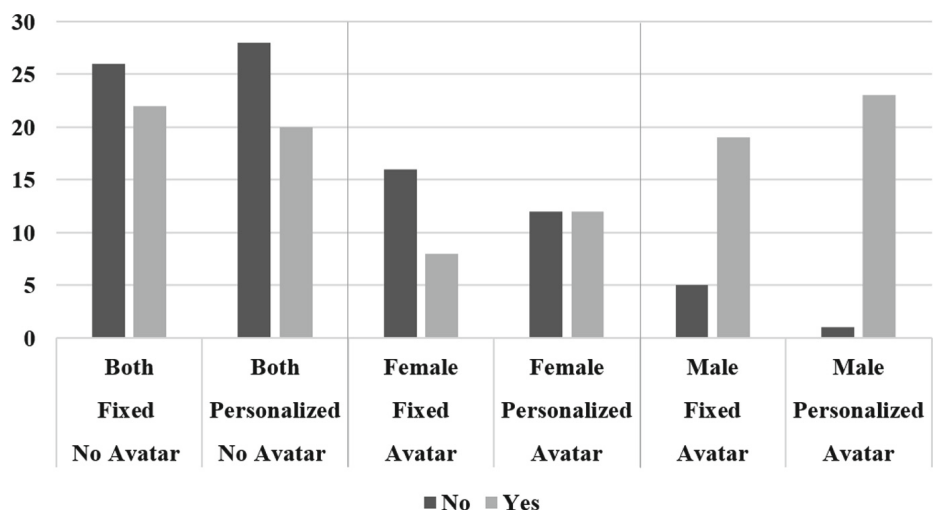

Fig. 3. Height comparison taking into account the gender differences

\subsection{Avatar Reports}

A chi-square test for association was conducted between avatar and the frequency with which participants placed the hand on top of the objects correctly. All expected cell frequencies were greater than five for the comparisons between: without an avatar and fixed height versus female participants with avatar and fixed height; without an avatar and fixed height versus male participants with avatar and fixed height; and without an avatar and personalised height versus female participants with avatar and personalised height. There was no statistically significant association between avatar and the frequency with which participants placed the hand on top of the objects correctly when comparing the groups without an avatar and fixed height versus female participants with avatar and fixed height, $\chi^{2}(1)=1.029, p=0.310$.

On the other hand, there was a statistically significant association between avatar and the frequency with which participants placed the hand on top of the objects correctly when comparing the groups without an avatar and fixed height versus male participants with avatar and fixed height, $\chi^{2}(1)=7.251, p=0.007$. There was a moderate association between avatar and the frequency with which participants placed the hand on top of the objects correctly, $\Phi=0.317, p=$ 0.007. The frequencies can be seen in Fig. 4.

There was no statistically significant association between avatar and the frequency with which participants placed the hand on top of the objects correctly when comparing the groups without an avatar and personalised height versus female participants with avatar and personalized height, $\chi^{2}(1)=0.450$, $p=0.502$.

Comparing participants without an avatar and personalised height vs male participants with avatar and personalised height, not all expected cell frequencies were greater than five. Therefore a Fisher's Exact test was conducted between avatar and the frequency with which participants placed the hand on top of the objects correctly. There was a statistically significant association between avatar 


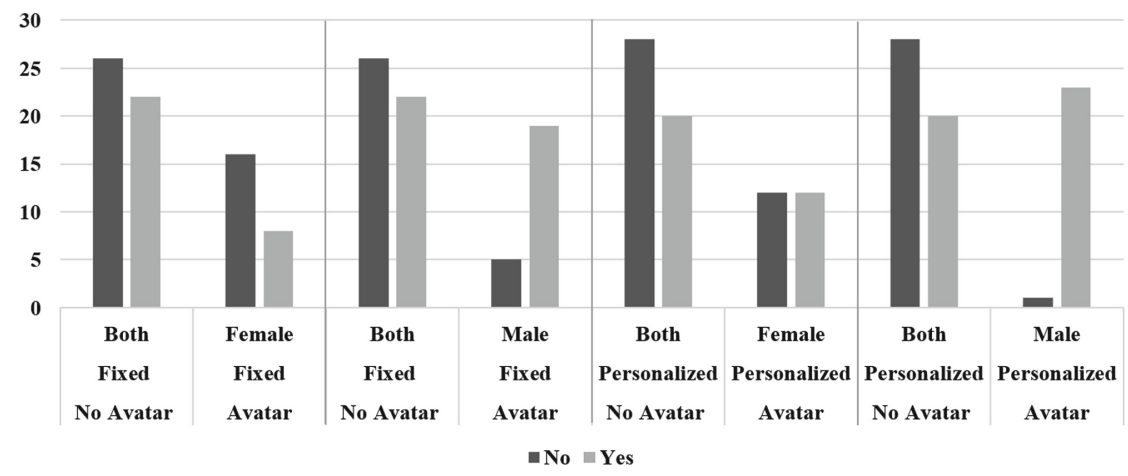

Fig. 4. Avatar comparison taking into account the gender differences.

and the frequency with which participants placed the hand on top of the objects correctly, $p<0.001$. The frequencies are shown in Fig. 4. Also, when participants could adjust their height to fell more natural, they tended to increase it, G2 $(\mathrm{M}=0.047$ and $\mathrm{SD}=0.085)$ and $\mathrm{G} 4(\mathrm{M}=0.022$ and $\mathrm{SD}=0.058)$.

\section{Discussion}

Having as motivation the little literature regarding the perception of small distances, the research team also studies how gender, height and avatar influences distance perception regarding those distances. Results of the Pearson's chi-square test for association found differences between gender for all the conditions with avatar present but no statistically significant differences in the absence of avatar. Results show that males have a higher rate than females when grabbing a real object through VE. One explanation for females underestimating distances can be because of the avatar's hand was not close enough, regarding representation, to their hand, causing perception changes when trying to grab the objects.

Regarding height, the Pearson's chi-square test for association shows that there is no statistically different accuracy between fixed and personalised height. An explanation would be that the changes in height made by the participants who had this possibility, was so insignificant that they made no difference when grabbing the real objects (changes in height in meters $\mathrm{M}=0.022$ and $\mathrm{SD}=0.058$ ).

As for the avatar variable, results of the Pearson's chi-square test for association show that there are statistically different results between both genders of the condition "fixed height with no avatar" and "males with fixed height and avatar". There were also statistically different results between both gender of the condition "personalized height with no avatar" and "males with personalized height and avatar". As for females, the presence of avatar did not make any difference. In the condition with an avatar, people tend to correct the hand position accordingly to the virtual representation, allowing males to increase their accuracy; but because females had perception changes, the presence of an avatar did not contribute to a better understanding of the distances. 


\section{Conclusion}

This work the impact of gender, avatar and height in distance perception of small distances in the VE. Results show that gender does make a difference in distance perception in some cases (any condition where an avatar was present). Moreover, results also evidentiate that height (Fixed or Personalized) did not make any statistical difference when grabbing objects through a VE, and the avatar (present or not present) made a difference in carrying out the task given.

This work is not free of limitations, one of the limitations is that one only has reports whether they grabbed the real object or not and not a quantitative value. We also need to evaluate further why the gender had differences in distance estimation. As for future work, one intends to evaluate small distance estimation with quantitative reports increasing the knowledge in this field. The research team also want to implement different hands for each gender and compare the new results with the results obtained in this work.

Acknowledgments. This work is financed by the ERDF - European Regional Development Fund through the Operational Programme for Competitiveness and Internationalisation - COMPETE 2020 Programme and by National Funds through the Portuguese funding agency, FCT - Fundação para a Ciência e a Tecnologia within project POCI-01-0145-FEDER-028618 entitled PERFECT - Perceptual Equivalence in virtual Reality For authEntiC Training.

\section{References}

1. Fuchs, P., Moreau, G., Guitton, P.: Virtual Reality: Concepts and Technologies, 1st edn. CRC Press, Boca Raton (2011)

2. Koutek, M.: Scientific Visualization in Virtual Reality: Interaction Techniques and Application Development. Delft University of Technology, mathesis, January 2003

3. OptiTrack: OptiTrack - Motion Capture (2018). http://optitrack.com/

4. Piryankova, I.V., de la Rosa, S., Kloos, U., Bülthoff, H.H., Mohler, B.J.: Egocentric distance perception in large screen immersive displays. Displays 34(2), 153-164 (2013)

5. Williams, B., Rasor, T., Narasimham, G.: Distance perception in virtual environments. In: Proceedings of the 6th Symposium on Applied Perception in Graphics and Visualization - APGV 2009, Chania, Crete, Greece, pp. 7-10. ACM Press (2009)

6. Bodenheimer, B., Meng, J., Wu, H., Narasimham, G., Rump, B., McNamara, T.P., Carr, T.H., Rieser, J.J.: Distance estimation in virtual and real environments using bisection. In: Proceedings of the 4th Symposium on Applied Perception in Graphics and Visualization - APGV 2007, Tubingen, Germany, pp. 35-40. ACM Press (2007)

7. Creem-Regehr, S.H., Kunz, B.R.: Perception and action. Wiley Interdisc. Rev. Cognitive Sci. 1(6), 800-810 (2010)

8. Mohler, B.J., Creem-Regehr, B.J., Thompson, W.B., Bülthoff, H.H.: The effect of viewing a self-avatar on distance judgments in an HMD-Based Virtual Environment. Presence Teleoperators Virtual Environ. 19(3), 230-242 (2010) 
9. Ries, B., Interrante, V., Kaeding, M., Phillips, L.: Analyzing the effect of a virtual avatar's geometric and motion fidelity on ego-centric spatial perception in immersive virtual environments. In: Proceedings of the 16th ACM Symposium on Virtual Reality Software and Technology - VRST 2009, Kyoto, Japan, pp. 59-66. ACM Press (2009)

10. McManus, E.A., Bodenheimer, B., Streuber, S., de la Rosa, S., Bülthoff, H.H., Mohler, B.J.: The influence of avatar (self and character) animations on distance estimation, object interaction and locomotion in immersive virtual environments. In: Proceedings of the ACM SIGGRAPH Symposium on Applied Perception in Graphics and Visualization - APGV 2011, Toulouse, France, pp. 37-44. ACM Press (2011)

11. Leyrer, M., Linkenauger, S.A., Bülthoff, H.H., Kloos, U., Mohler, B.: The influence of eye height and avatars on egocentric distance estimates in immersive virtual environments. In: Proceedings of the ACM SIGGRAPH Symposium on Applied Perception in Graphics and Visualization - APGV 2011, Toulouse, France, pp. 67-74. ACM Press, August 2011

12. Coluccia, E., Louse, G.: Gender differences in spatial orientation: a review, vol. 24, no. 3, pp. 329-340. http://www.sciencedirect.com/science/article/pii/ S0272494404000477

13. Naceri, A., Chellali, R.: The effect of isolated disparity on depth perception in real and virtual environments. In: 2012 IEEE Virtual Reality (VR). IEEE, March 2012

14. Creem-Regehr, S.H., Willemsen, P., Gooch, A.A., Thompson, W.B.: The influence of restricted viewing conditions on egocentric distance perception: implications for real and virtual indoor environments. Perception 34(2), 191-204 (2005)

15. Peer, A., Ponto, K.: Preliminary exploration: perceived egocentric distance measures in room-scale spaces using consumer-grade head mounted displays. In: IEEE Virtual Reality (VR), Los Angeles, CA, USA, pp. 275-276. IEEE, March 2017

16. Renner, R.S., Velichkovsky, B.M., Helmert, J.R.: The perception of egocentric distances in virtual environments - a review. ACM Comput. Surv. 46(2), 1-40 (2013)

17. Li, Z., Phillips, J., Durgin, F.H.: The underestimation of egocentric distance: evidence from frontal matching tasks. Attention, Percept. Psychophysics 73(7), 2205$2217(2011)$ 